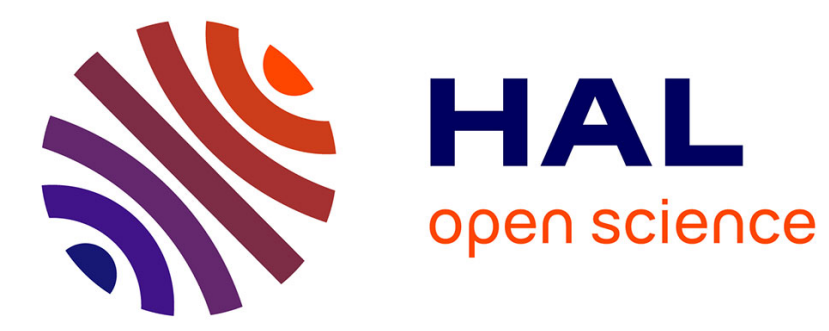

\title{
Testing the assumptions behind importance sampling
}

Siem Jan Koopman, Neil Shephard, Drew Creal

\section{To cite this version:}

Siem Jan Koopman, Neil Shephard, Drew Creal. Testing the assumptions behind importance sampling.

Econometrics, 2009, 149 (1), pp.2. 10.1016/j.jeconom.2008.10.002 . hal-00581879

\section{HAL Id: hal-00581879 \\ https://hal.science/hal-00581879}

Submitted on 1 Apr 2011

HAL is a multi-disciplinary open access archive for the deposit and dissemination of scientific research documents, whether they are published or not. The documents may come from teaching and research institutions in France or abroad, or from public or private research centers.
L'archive ouverte pluridisciplinaire HAL, est destinée au dépôt et à la diffusion de documents scientifiques de niveau recherche, publiés ou non, émanant des établissements d'enseignement et de recherche français ou étrangers, des laboratoires publics ou privés. 


\section{Accepted Manuscript}

Testing the assumptions behind importance sampling

Siem Jan Koopman, Neil Shephard, Drew Creal

PII:

S0304-4076(08)00169-3

DOI:

10.1016/j.jeconom.2008.10.002

Reference: ECONOM 3116

To appear in: Journal of Econometrics

Please cite this article as: Koopman, S.J., Shephard, N., Creal, D., Testing the assumptions behind importance sampling. Journal of Econometrics (2008),

doi:10.1016/j.jeconom.2008.10.002

This is a PDF file of an unedited manuscript that has been accepted for publication. As a service to our customers we are providing this early version of the manuscript. The manuscript will undergo copyediting, typesetting, and review of the resulting proof before it is published in its final form. Please note that during the production process errors may be discovered which could affect the content, and all legal disclaimers that apply to the journal pertain. 


\title{
Testing the assumptions behind importance sampling
}

\author{
SIEM JAN KOOPMAN* \\ Department of Econometrics, Vrije Universitiet Amsterdam \\ NEIL SHEPHARD ${ }^{\dagger}$ \\ Oxford-Man Institute, University of Oxford \\ DREW CREAL ${ }^{\ddagger}$ \\ Department of Econometrics, Vrije Universitiet Amsterdam
}

August 2008

\begin{abstract}
Importance sampling is used in many areas of modern econometrics to approximate unsolvable integrals. Its reliable use requires the sampler to possess a variance, for this guarantees a square root speed of convergence and asymptotic normality of the estimator of the integral. However, this assumption is seldom checked. In this paper we use extreme value theory to empirically assess the appropriateness of this assumption. Our main application is the stochastic volatility model, where importance sampling is commonly used for maximum likelihood estimation of the parameters of the model.
\end{abstract}

Keywords: Extreme value theory; Importance sampling; Simulation; Stochastic volatility.

JEL classification codes: C10, C22, C32, C51

\section{Introduction}

This paper develops tools for evaluating the importance sampling techniques that are popular in econometrics. There are two situations where importance sampling is commonly applied. It is often used to numerically evaluate the likelihood function in missing data models, when an analytic expression for the likelihood function does not exist. This is of primary importance in the literature on maximum likelihood estimation of stochastic volatility (SV) models. Importance sampling is also popular in Bayesian applications when computing expectations of the posterior distribution.

In a fundamental contribution, Geweke (1989) argued that importance sampling should only be used in settings where the variance of the importance weights is known to exist. Failure of this condition can lead to slow and unstable convergence of the estimator as the central limit

${ }^{*}$ Corresponding author. Address: De Boelelaan 1105, NL-1081 HV Amsterdam; Tel: +31 20598 6019; Fax: +31 20598 6020; Email: s.j.koopman@feweb.vu.nl

†Address: Blue Boar Court, 9 Alfred Street, Oxford OX1 4EH, UK; Email: neil.shephard@economics.ox.ac.uk

${ }^{\ddagger}$ Address: De Boelelaan 1105, NL-1081 HV Amsterdam; Email: dcreal@feweb.vu.nl 
theorem governing convergence fails to hold. Robert and Casella (2004, p.90) provide detailed examples of importance samplers that fail this condition and note that ignoring the problem can result in strongly biased estimators. While the variance conditions can be checked analytically in low dimensional problems, proving that they are met in high dimensions can be challenging. Many econometrics and statistics papers have recently been written which, in effect, a priori assume that this condition holds. This is the case in the stochastic volatility literature; see, e.g., Hendry and Richard (1991), Danielsson and Richard (1993), Sandmann and Koopman (1998), Elerian, Chib, and Shephard (2001), Durham and Gallant (2002), Durham (2006), and Asai and McAleer (2006). It is also assumed to hold in the macroeconomics literature on dynamic stochastic general equilibrium models; see, e.g. An and Schorfheide (2007).

In this paper, we develop methods to check for the existence of the variance of the importance weights. This will be based on the application of extreme value theory. ${ }^{1}$ Limit theorems from extreme value theory imply that we can learn about the variance of the weights by studying the behavior of their distribution in the right hand tail. Earlier work on testing whether the variance of the importance weights exists also using extreme value theory was conducted by Monahan (1993, 2001). Monahan examined the tail-behavior of the weights by building a test statistic from the Hill (1975) estimator. We take an alternative approach and develop three different test statistics using the well-known principles of the Wald, score and likelihood ratio tests. We also propose graphical diagnostics for a complete insight.

The new test procedures are carried out for two Monte Carlo studies and for the maximum likelihood analysis of two SV models. The latter models have been our main motivation to investigate the problem in detail. It will be shown that sometimes the variance of the importance weights does not exist when estimating parameters of SV models. Although our main focus will be on likelihood estimation, our methods apply generally to all applications of importance sampling including Bayesian approaches.

A review of importance sampling is provided in Section 2. In Section 3, we discuss the statistical estimation of tail indexes for extremes and introduce our test statistics. Section 4 includes results from some Monte Carlo experiments that are conducted to evaluate the power and size of the proposed tests. We apply the test statistics and graphical diagnostics to maximum likelihood estimation of the parameters of two SV models in Section 5. Section 6 concludes.

\footnotetext{
${ }^{1}$ We should note that extreme value theory has been applied in the context of financial economics and insurance in order to determine the thickness of the tail of financial returns. Furthermore, it is used to compute various measures of risk. References to this literature include Embrechts, Kluppelberg, and Mikosch (1997, Section 6.5.2) and Danielsson and de Vries (1997). The application of the theory is much harder in that context for the i.i.d. assumption does not hold.
} 


\section{Importance sampling}

Importance sampling was discussed as early as Kahn and Marshall (1953) and Marshall (1956), while it was popularised in the influential monograph by Hammersley and Handscomb (1964, Section 5.4). It was first used in econometrics by Kloek and van Dijk (1978) in their work on computing posterior densities. Consider an integral that cannot be solved analytically

$$
\mathcal{I}=\int_{\mathcal{X}} h(x) f(x) d x,
$$

where $h(x)$ is a function and $f(x)$ is a probability density. An importance sampling density $g(x)$ is selected from which it is easy to simulate and whose support is also $\mathcal{X}$. The integral $\mathcal{I}$ can be approximated by

$$
\widehat{\mathcal{I}}=\frac{1}{N} \sum_{i=1}^{N} w_{i}, \quad \text { where } \quad w_{i}=w\left(x^{i}\right),
$$

for a large number of $N$ draws and with

$$
w(x)=\frac{h(x) f(x)}{g(x)} \text { and } \quad x^{i} \sim g(x) .
$$

The strong law of large numbers implies that $\widehat{\mathcal{I}}$ will be a consistent estimator of $\mathcal{I}$ without any additional assumptions. By construction we know that the $\left\{w_{i}\right\}_{i=1}^{N}$ are i.i.d. and that $\mathbb{E}[w(x)]=\mathcal{I}$. As a result, a simple application of Kolmogorov's strong law of large numbers (e.g. Geweke (1989) and Geweke (2005, p. 114)) shows that

$$
\widehat{\mathcal{I}} \stackrel{a . s .}{\rightarrow} \mathcal{I}, \quad \text { as } N \rightarrow \infty,
$$

whatever importance sampler we design. In practice, the normalizing constant of $f(x)$ or $g(x)$ in (2) may not be known requiring a self-normalized importance sampling estimator. ${ }^{2}$

In order to easily measure the precision of $\widehat{\mathcal{I}}$ and to guarantee that the rate of convergence to $\mathcal{I}$ is $N^{1 / 2}$, it is helpful to have a Gaussian central limit theorem for $\widehat{\mathcal{I}}$. We know that a necessary condition for the application of the Lindeberg-Lévy central limit theorem is the existence of a variance for the importance weights. This would allow us to conclude that

$$
\sqrt{N}(\widehat{\mathcal{I}}-\mathcal{I}) \stackrel{d}{\rightarrow} N\left(0, \gamma^{2}\right) .
$$

Expressions for the asymptotic variance $\gamma^{2}$ of the importance sampling estimator can be found in Geweke (2005, p. 114) and Cappé, Moulines, and Rydén (2005, p. 287). However, the existence of the variance of the importance weights is by no means guaranteed. If it does not exist, the importance sampling estimator may convergence erratically making estimates unreliable. An approach for testing for the existence of the variance is considered in the next section.

\footnotetext{
${ }^{2}$ This self-normalized estimator induces a small bias but it remains consistent. Liu (2001, p. 35) and Robert and Casella (2004, p.95) note that the self-normalized estimator may be preferred as it typically has smaller mean square error than the unbiased estimator.
} 


\section{$3 \quad$ Extreme value theory and inference}

\subsection{Extreme value theory}

We begin this section briefly reviewing the results from extreme value theory that are relevant for this paper; see Embrechts, Kluppelberg, and Mikosch (1997) for a detailed discussion. Assume we have a sequence $w_{1}, w_{2}, \ldots, w_{N}$ of $i . i . d$ random variables from a distribution function $F$. The $\left\{w_{i}\right\}_{i=1}^{N}$ need not represent importance weights, although that will be the case in our work below. Denote the endpoint of the distribution function $F$ as $w_{E}=\sup \{w: F(w)<1\} \leq+\infty$. Pickands (1975) defined a new sequence of $n$ random variables $Z_{1}, \ldots, Z_{n}$ created from those $w_{i}$ that lie above a threshold $u$, i.e. $Z_{i}=w_{i}-u$ where $n \leq N$. Pickands (1975) proved that as $N$ and $u$ get larger the distribution of the excesses $\left\{Z_{i}\right\}_{i=1}^{n}$ is well approximated by the generalized Pareto distribution. More formally, he showed that

$$
\lim _{u \rightarrow w_{E}} \sup _{0<z<w_{E}-u}\left|F_{u}(z)-G P D(z ; \xi, \beta)\right|=0,
$$

where $F_{u}(z)$ is the distribution function of the excesses conditional on threshold $u$ and $w>u$. $G P D$ denotes the generalized Pareto distribution with density

$$
f(z ; \xi, \beta)=\frac{1}{\beta}\left(1+\xi \frac{z}{\beta}\right)^{-\frac{1}{\xi}-1}, \quad z \in D(\xi, \beta)>u, \quad \beta>0
$$

Here

$$
D(\xi, \beta)= \begin{cases}{[0, \infty),} & \xi \geq 0, \\ {[0,-\beta / \xi],} & \xi<0 .\end{cases}
$$

The threshold $u$ plays a crucial role in this result and its selection will be discussed in Section 4. We continue by assuming that $u$ is a given threshold.

The shape parameter $\xi$ of the generalized Pareto distribution characterizes the thickness of the distribution's tails. In the context of extreme value theory, the value of $\xi$ is determined by the thickness of the tails of $F$. This means that we can extract information about the thickness of the tails of the importance weights by making inference on the shape parameter using the excesses $\left\{Z_{i}\right\}_{i=1}^{n}$. In fact, it can be shown that when $\xi>0$ then $\mathbb{E}\left[w^{j}\right]=\infty$ for $j \geq 1 / \xi$. Consequently, the number of moments that the distribution of the importance weights has can be determined by focusing on $\xi$. In particular, we are interested in establishing that $\xi \leq 1 / 2$.

We know by construction of the importance sampler that $\xi \leq 1$. The cases of $\xi<0$ deal with situations where the $\left\{w_{i}\right\}_{i=1}^{N}$ have some upper bound. This is of some relevance in importance sampling in the case of the sampler being bounded, that is

$$
f(x) \leq M g(x),
$$


for some finite choice of $M>0$. Then if (4) is true for $x \in \mathcal{X}$, the support of the importance weights $\left\{w_{i}\right\}_{i=1}^{N}$ will be bounded and the existence of all moments is guaranteed. This is, in practice, quite unusual. In most cases of real interest, the sampler is not bounded, so $\xi \in[0,1]$.

\subsection{Previous work: Monahan's test}

Monahan $(1993,2001)$ proposed testing if an importance sampler's weights have a finite variance using the Hill (1975) estimator. The Hill estimator is an alternative to the generalized Pareto distribution for estimating the tail parameter $\xi$. It is defined as

$$
\xi^{H i l l}=\frac{1}{k} \sum_{j=1}^{k} \log w_{(N-j+1)}-\log w_{(N-k)},
$$

where $w_{(1)} \leq w_{(2)} \leq \ldots \leq w_{(N)}$ are the order statistics from a sample of importance weights. The tuning parameter $k$ determines the number of large order statistics used in (5) meaning that it plays a similar role as the threshold parameter $u$ above.

The Hill estimator is largely regarded as a nonparametric estimator although it can be interpreted as a maximum likelihood estimator under appropriate conditions on the distribution function $F$. Asymptotic normality of the Hill estimator has been studied by Haeusler and Teugels (1985) who have shown that it depends on the rate of growth of $k$ relative to $N$. Monahan used their result

$$
\sqrt{k / \xi^{2}}\left(\widehat{\xi}^{\text {Hill }}-\xi\right) \stackrel{d}{\rightarrow} N(0,1),
$$

and chose the values $k=2 N^{1 / 3}$ and $k=4 N^{1 / 3}$ to build a test statistic. Monahan's test has as its null hypothesis that the variance of the importance weights is infinite making it a test of $H_{0}: \xi \geq 1 / 2$ versus $H_{1}: \xi<1 / 2$. In Section 4, we compare Monahan's test with the tests we propose below. To make the comparison easier, we have reversed the null hypothesis of his test to make it consistent with ours.

\subsection{Proposed methods}

We propose testing for an infinite variance by constructing test statistics from the maximum likelihood estimates of the generalized Pareto distribution. Our paper builds on the work of Smith $(1985,1987)$ who studied the properties of the maximum likelihood estimator of $\theta=(\xi, \beta)^{\prime}$ in this setting. We construct three test statistics using his results. Formally, the hypotheses we will be interested in deciding between is

$$
H_{0}: \xi \leq \frac{1}{2}, \quad \text { and } \quad H_{1}: \xi>\frac{1}{2} .
$$

The null hypothesis implies the existence of the variance while the alternative denies it. In practice it is helpful to make the null a point hypothesis (see, for example, Cox and Hinkley 
(1974, pp. 331-334)), so making the comparison between

$$
H_{0}: \xi=\frac{1}{2}, \quad \text { and } \quad H_{1}: \xi>\frac{1}{2} \text {. }
$$

As we have a parametric model for the weights over a threshold, we will use a likelihood function to carry out the testing. This approach to inference is often called the peaks over threshold method. We will study the behavior of the score and likelihood ratio tests of the hypothesis.

\subsection{Estimation and Wald test}

Starting with $N$ importance weights, we first find the $n$ importance weights that lie above the threshold and we construct the excesses $z_{1}, \ldots, z_{n}$ from these large importance weights. The log-likelihood for a sample $z_{1}, \ldots, z_{n}$ over the threshold $u$ equals

$$
\log f(z ; \theta)=-n \log \beta-\left(1+\frac{1}{\xi}\right) \sum_{i=1}^{n} \log \left(1+\xi \beta^{-1} z_{i}\right) .
$$

Maximum likelihood estimation of the parameter vector $\theta=(\xi, \beta)^{\prime}$ is discussed in Smith (1985, 1987). The asymptotic distribution of the maximum likelihood estimator $\hat{\theta}$ is given by

$$
\sqrt{n}(\widehat{\theta}-\theta) \stackrel{d}{\rightarrow} N\left(0, \mathbf{I}(\theta)^{-1}\right) \quad \text { where } \quad \mathbf{I}(\theta)^{-1}=(1+\xi)\left(\begin{array}{cc}
2 \beta^{2} & \beta \\
\beta & 1+\xi
\end{array}\right),
$$

where $n \mathbf{I}(\theta)$ denotes the expected information matrix. Smith (1985) has shown that likelihood inference is standard for this problem as long as $\xi>-1 / 2$. This covers the null hypothesis value $H_{0}: \xi=\frac{1}{2}$ and all the values under the alternative. Davison and Smith (1990) describe how to reduce maximum likelihood estimation of (7) to a one-dimensional parameter search. Substituting $\beta=\xi / \tau$ into (7) and differentiating with respect to $\xi$ and $\tau$ produces

$$
\begin{aligned}
& \xi=\xi(\tau)=\frac{1}{n} \sum_{i=1}^{n} \log \left(1+\tau z_{i}\right), \\
& \frac{n}{\tau}=\left(1+\frac{1}{\xi(\tau)}\right) \sum_{i=1}^{n} \frac{z_{i}}{1+\tau z_{i}} .
\end{aligned}
$$

Equation (9) can then be substituted into (10) and solved numerically for an estimate $\hat{\tau}$. Given $\hat{\tau}$, the maximum likelihood estimates $\hat{\xi}$ and $\hat{\beta}$ are easily obtained. A sensible starting value for $\tau=\xi / \beta$ can be found by imposing the null hypothesis $\xi=0.5$ and noting that the expected value of $Z$ is

$$
\mathbb{E}[Z]=\frac{\beta}{\xi^{2}} \frac{\Gamma\left(\xi^{-1}-1\right)}{\Gamma\left(1+\xi^{-1}\right)}=2 \beta, \quad \text { for } \xi=0.5
$$

Iterations can therefore start at $\tau=1 / \bar{z}$ where $\bar{z}=n^{-1} \sum_{i=1}^{n} z_{i}$. 
Once the maximum likelihood estimator of $\theta$ is found the Wald test can be computed to test the null hypothesis (6). As an alternative to the Wald test formulation, we can consider the $t$-statistic

$$
t=\sqrt{\frac{n}{3 \hat{\beta}^{2}}}\left(\hat{\xi}-\frac{1}{2}\right),
$$

where $\hat{\beta}$ is the maximum likelihood estimate of $\beta$. The null hypothesis is rejected when the $t$-statistic takes a large positive value compared to a standard normal random variable.

\subsection{Estimation under null hypothesis and score test}

The maximum likelihood estimator of $\beta$ under the null hypothesis of $\xi=0.5$ can be found by univariate Fisher scoring. The score value of $\beta$ is given by

$$
\begin{aligned}
s_{r}^{\beta} & =-\frac{n}{\beta}+\frac{3}{2} \beta^{-2} \sum_{i=1}^{n} \frac{z_{i}}{1+0.5 \beta^{-1} z_{i}}, \\
& =n \beta^{-1}\left(\frac{3}{n} \sum_{i=1}^{n} \frac{z_{i}}{2 \beta+z_{i}}-1\right),
\end{aligned}
$$

Using the results in (11) once again, we can take $0.5 \bar{z}$ as the initial value for $\beta$. After convergence the restricted estimate of $\beta$ is obtained which we denote by $\hat{\beta}_{r}$.

The one-sided score statistic will be used for testing the null hypothesis as this will be computationally simple. It is based on the score value of $\xi$ under the null hypothesis and is given by

$$
\begin{aligned}
s_{r}^{\xi} & =4 \sum_{i=1}^{n} \log \left(1+0.5 \beta^{-1} z_{i}\right)-3 \beta^{-1} \sum_{i=1}^{n} \frac{z_{i}}{1+0.5 \beta^{-1} z_{i}} \\
& =4 \sum_{i=1}^{n} \log \left(1+\frac{z_{i}}{2 \beta}\right)-\frac{6}{n} \sum_{i=1}^{n} \frac{z_{i}}{2 \beta+z_{i}}, \quad \text { for } \xi=0.5 .
\end{aligned}
$$

This score value for the null hypothesis (6) is a function of $\beta$ and it can be evaluated when $\beta$ is replaced by its (restricted) maximum likelihood estimator $\hat{\beta}_{r}$. We know from the results of Smith $(1985,1987)$ that

$$
s_{*}^{\xi}=\frac{1}{\sqrt{2 n}} s_{r}^{\xi} \stackrel{d}{\rightarrow} N(0,1),
$$

which provides a simple test. We reject the null $H_{0}: \xi=\frac{1}{2}$ when $\hat{s}_{*}^{\xi}$ is significantly positive and where $\hat{s}_{*}^{\xi}$ is $s_{*}^{\xi}$ with $\beta$ replaced by $\hat{\beta}_{r}$.

\subsection{Likelihood ratio test}

When estimation of $\theta$ has taken place under the constraint that $\xi \geq 0.5$, to deliver $\widetilde{\theta}$, together with estimation under the null hypothesis, the likelihood ratio statistic can also be used to test 
the null

$$
L R=2\left\{\log f(z ; \widetilde{\theta})-\log f\left(z ; \hat{\beta}_{r}, \xi=0.5\right)\right\} .
$$

Of course, the null is on the boundary next to the alternative and so the limiting distribution of the statistic is

$$
L R \stackrel{d}{\rightarrow} 0.5\left(\chi_{0}^{2}+\chi_{1}^{2}\right), \quad \text { under } H_{0}
$$

The $\chi_{0}^{2}$ distribution is a degenerate distribution with all its probability mass at zero. There exists a 0.5 probability that the likelihood ratio statistic will be zero (as $\widehat{\xi}$ will be negative). The rest of the time the statistic will have a $\chi_{1}^{2}$ distribution (e.g. Chernoff (1954) and Gourieroux, Holly, and Monfort (1981)).

\section{Monte Carlo evidence}

To check the effectiveness of extreme value theory in practice for testing the assumptions behind importance sampling we consider two experiments. The first is based on sampling from a normal density to approximate a normal density with a different variance. Our second experiment is the classic example of estimating a Student's $t$ distribution using a normal candidate density.

\subsection{Normal density experiment}

We take $f(x)$ to be the normal density $N(0,1)$ and the importance sampling density $g(x)$ is $N\left\{0,(1+\epsilon)^{-1}\right\}$ where $\epsilon$ is a positive number. The function $h(x)$ is constant and equal to one. Thus for this problem $\mathcal{I}=1$ in (1). The question is whether the variance of the importance weights exists? The weight function is given by

$$
w(x)=\frac{h(x) f(x)}{g(x)}=\frac{1}{\sqrt{1+\epsilon}} \exp \left(\frac{\epsilon}{2} x^{2}\right),
$$

which obviously has $\mathbb{E}[w(x)]=1$, while

$$
\begin{aligned}
\mathbb{E}\left[w(x)^{2}\right] & =\frac{1}{\sqrt{2 \pi}} \frac{\sqrt{1+\epsilon}}{1+\epsilon} \int_{-\infty}^{\infty} \exp \left(\epsilon x^{2}\right) \exp \left[-\frac{1}{2} x^{2}(1+\epsilon)\right] d x \\
& =\frac{1}{\sqrt{2 \pi}} \frac{1}{\sqrt{1+\epsilon}} \int_{-\infty}^{\infty} \exp \left[-\frac{1}{2} x^{2}(1-\epsilon)\right] d x,
\end{aligned}
$$

which is bounded only if $\epsilon<1$. The variance of the importance weights will not exist if $\epsilon \geq 1$, meaning that the central limit theorem will not hold for importance samplers built in that range of values.

\section{$<$ INSERT TABLE 1 HERE>}

In Table 1, we present the diagnostic tests for a large number of different thresholds. They show that for small values of $\epsilon$ importance sampling is valid while for values of $\epsilon \geq 1$ the variance 
of the importance weights does not exist. For our test statistics to be effective, we require a simulation sample size $N$ that is sufficiently large (say, 100,000). The Wald test appears to have the most power. It can potentially be oversized if the threshold is selected too high. It can also perform poorly as the threshold gets extremely small. Tests that are slightly oversized are not necessarily problematic. Rejecting importance samplers whose weights are barely finite may not be a bad practice as these algorithms can still be unstable. The $L R$ statistic has nearly the same power and it behaves more robustly for different values of $u$ than the Wald statistic. This implies that computing more than one statistic is a good idea. Both of these statistics are considerably more powerful than the Monahan statistic computed using either $k=4 N^{1 / 3}$ or $k=2 N^{1 / 3}$. We note that the Monahan statistic performs better than our tests when the number of draws $N$ is small (e.g. $N \leq 5,000)$.

We have chosen the threshold $u$ in an arguably ad hoc manner to both facilitate the Monte Carlo study and to understand how different values of $u$ impact the tests. In practice, the threshold can be chosen in alternative ways and users may want to compare different selection methods. Methods that are common in the extreme value literature are a mean residual life plot or by minimizing the distance between the empirical distribution function and the generalized Pareto distribution evaluated at the maximum likelihood estimates; see, e.g. Embrechts, Kluppelberg, and Mikosch (1997) and Gonzalo and Olmo (2004). We recommend graphing the maximum likelihood estimates of $\xi$ along with confidence bands for a large number of thresholds (say 50 values from $0.01 N$ to $0.50 N$ ). We illustrate this strategy in our application to the stochastic volatility model in Section 5 .

Our proposed test statistics do not provide strict guarantees for a successful importance sampling procedure. We believe that graphical diagnostics, although informal, can play a complementary role. They are a simple way to detect severely deficient importance sampling algorithms. A graph of the largest 100 weights indicates the seriousness of the outliers. The remaining weights can be represented via a histogram. Plotting estimates of the variance of the weights recursively can show the impact that each weight has on the estimate. As the sample increases, the variance should converge to a constant if it exists. Plots like these do not always detect problems and we recommend viewing them for many different sequences of random numbers.

\section{$<$ INSERT FIGURE 1 HERE $>$}

Graphs for the normal density experiment with $\epsilon=0.5,1.0$, and 1.2 are presented in the three rows of Figure 1. The values of $\epsilon$ were chosen to compare the output from importance samplers representing both the null and alternative hypotheses. When $\epsilon=0.5$, the variance 
is finite and the recursive estimate of the variance convergences smoothly. The graphs for the cases $\epsilon=1.0$ and 1.2 are clearly informative in this example. The large jumps in the recursive variance estimates correspond to the outliers in the left hand graphs of the middle and bottom rows.

\subsection{Normal approximation to the Student's $t$ distribution}

$$
<\text { INSERT TABLE } 2 \text { HERE }>
$$

Our second Monte Carlo experiment is the classic example of approximating a Student's $t$ distribution with a normal distribution. This example can be used to evaluate the power of the tests. A similar experiment was also considered by Monahan (1993). We let $f(x)$ be a Student's $t$ distribution with $\nu$ degrees of freedom and where $g(x)$ is a normal density with mean zero and variance $\nu /(\nu+1) \cdot{ }^{3}$ Once again the function $h(x)$ is constant and equal to one. The weight function is simply

$$
w(x)=\frac{\Gamma\left(\frac{\nu+1}{2}\right) \sqrt{2}}{\Gamma\left(\frac{\nu}{2}\right) \sqrt{\nu+1}} \frac{\left(1+x^{2} / \nu\right)^{-(\nu+1) / 2}}{\exp \left(-0.5\left(\frac{\nu+1}{\nu}\right) x^{2}\right)} .
$$

In this example, the quantity $\mathbb{E}\left[w(x)^{2}\right]$ does not exist.

Table 2 contains the diagnostic tests for different values of the degrees of freedom and the threshold. The performance of the tests are mostly in agreement with the results from the normal experiment above. The Wald test is the most powerful and performs the best when $N$ is reasonably large. The difference in power between this test and the others is significant when $\nu \geq 20$. Figure 2 plots the series of graphical diagnostics for two values of the degrees of freedom. The recursive variance estimates easily detect problems when $\nu=5$ while $\nu=20$ also appears doubtful. Problems created by large values of $\nu$ can be hard to recognize with plots, making the combination of test statistics and graphical diagnostics valuable.

\section{$<$ INSERT FIGURE 2 HERE $>$}

\section{An illustration: stochastic volatility model}

Stochastic volatility (SV) models have gained considerable interest in econometrics as a class of models which allows volatility to change through time. These models are often stated in continuous time and appear frequently in the option pricing literature. Ghysels, Harvey, and Renault (1996) and Shephard (2005) review the literature on this topic from an econometric viewpoint. The likelihood function for SV models is an integral than cannot be solved analytically but it can

\footnotetext{
${ }^{3}$ We believe we have implemented the same experiment as Monahan. A potential missprint in his paper may imply an alternative normal importance density with variance $(\nu+1) / \nu$ rather than the importance density with variance $\nu /(\nu+1)$ given here.
} 
be evaluated by Monte Carlo simulation. Papers that carry out maximum likelihood analysis of SV models using importance sampling include Danielsson (1994), Shephard and Pitt (1997), Durbin and Koopman (1997), Sandmann and Koopman (1998), Durham and Gallant (2002), Durham (2003), Liesenfeld and Richard (2003), Brandt and Kang (2004), Durham (2006), Asai and McAleer (2006), Richard and Zhang (2007), and Jungbacker and Koopman (2007).

The SV model we use to illustrate our discussion will be a discrete time, log-normal, univariate SV model for a sequence of returns $y_{t}$ for $t=1,2, \ldots, T$. It can be thought of as an Euler discretisation of a continuous time SV model where the spot volatility follows a log-normal Ornstein-Uhlenbeck process (e.g. Hull and White (1987)). It is given by

$$
\begin{aligned}
& y_{t}=\mu_{y}+\exp \left(\alpha_{t} / 2\right) \varepsilon_{t}, \\
& \alpha_{t+1}=\mu_{\alpha}+\phi\left(\alpha_{t}-\mu_{\alpha}\right)+\sigma_{\eta} \eta_{t}, \quad \alpha_{1} \sim N\left\{0, \sigma_{\eta}^{2} /\left(1-\phi^{2}\right)\right\},
\end{aligned}
$$

where the disturbances $\varepsilon_{t}$ and $\eta_{t}$ are independent of $\alpha_{t}$ for $t=1, \ldots, T$. The unconditional mean of the returns is given by $\mu_{y}$ while the mean of the log-volatility is $\mu_{\alpha}$. The degree of volatility persistence is measured by the autoregressive parameter $\phi$ and the parameter $\sigma_{\eta}$ is the standard deviation of the disturbances to the log-volatility process. We will consider two cases for $\varepsilon_{t}$. The first model, denoted by SV- $N$, assumes that $\varepsilon_{t} \sim N(0,1)$ while the second model, denoted SV-t, has $\varepsilon_{t} \sim t_{\nu}$ where $\varepsilon_{t}$ is scaled to have unit variance.

Both SV models require the estimation of the parameter vector $\Theta$. Inference for the unknown parameters will be based on $f(y ; \Theta)$ where $y=\left(y_{1}, \ldots, y_{T}\right)^{\prime}$. Clearly

$$
f(y ; \Theta)=\int_{\mathcal{X}^{T}} f(y \mid \alpha ; \Theta) f(\alpha ; \Theta) d \alpha,
$$

where $\alpha=\left(\alpha_{1}, \ldots, \alpha_{T}\right)^{\prime}$. The analytic form of $f(y \mid \alpha ; \Theta)$ and $f(\alpha ; \Theta)$ can be deduced from (14), but the integral in (15) can only be solved numerically due to the non-Gaussian nature of the model. Our approximation of $f(y ; \Theta)$ by importance sampling is given by

$$
\widehat{f(y ; \Theta)}=\frac{1}{N} \sum_{i=1}^{N} w_{i}, \quad \text { where } \quad w_{i}=w\left(\alpha^{i}\right)
$$

with

$$
w(\alpha)=\frac{f(y \mid \alpha ; \Theta) f(\alpha ; \Theta)}{g(\alpha \mid y ; \Theta)} \quad \text { and } \quad \alpha^{i} \sim g(\alpha \mid y ; \Theta)
$$

It is assumed that $g(\alpha \mid y ; \Theta)$ is strictly positive for all $\alpha \in \mathcal{X}^{T}$. We take the importance sampler $g(\alpha \mid y ; \Theta)$ as the Laplace approximation to the posterior of $\alpha_{1}, \ldots, \alpha_{T}$ given the data $y_{1}, \ldots, y_{T}$; see, e.g., Shephard and Pitt (1997) and Robert and Casella (2004, p. 107). The initial value of $\alpha_{1}$ is a draw from the unconditional distribution of $\alpha_{t}$. The maximum likelihood estimator of $\Theta$ is referred to as $\widehat{\Theta}$. When maximising the log-likelihood function, we use $N=3,000$ draws where the same underlying uniform random variables are used for each likelihood evaluation. 
In our empirical study we use the return series $y_{t}$ of daily Standard \& Poor's 500 stock index closures. The historical return series is for the period 3rd January 1985 to 31st July 2007 and was obtained from Yahoo Finance. Our sample consists of 5, 693 daily observations. Importance sampling is being carried out over 5,693 dimensions in this case. The continuously compounded (raw) returns on the stock index are not adjusted for dividends and they are expressed in percentage terms and therefore given by $y_{t}=100\left(\ln P_{t}-\ln P_{t-1}\right)$ where $P_{t}$ denotes the closing price of the Standard \& Poor's 500 index on day $t$.

\section{$<$ INSERT TABLE 3 HERE >}

\subsection{Test statistics and graphical diagnostics}

We start by considering the SV-N model whose estimated parameters are reported in Table 3 together with their $95 \%$ confidence intervals. We estimated both models on two different sample sizes, the full sample and a smaller sample corresponding to 2nd January 2002 through 31st July 2007. To illustrate our diagnostic procedure, we take the simulated maximum likelihood estimate $\Theta=\widehat{\Theta}$ as fixed. Conditional on this estimate, we increase the simulation size from $N=3,000$ to $N^{+}=100,000$ and produce a set of diagnostic graphs. The question we ask is if the variance of the weights exists for $\Theta=\widehat{\Theta}$, basing the diagnostic graphics on these i.i.d. 100, 000 draws. The largest 100 importance weights are presented in Figure 3 together with a histogram of the remaining 99, 900 smaller weights. The third graph presents recursive estimates of the variance of the importance weights. The final graph presents estimates of $\xi$ along with an asymmetric $95 \%$ confidence interval for different thresholds $u$ ranging from the largest 1 percent to 50 percent of the $N^{+}$importance weights.

\section{$<$ INSERT FIGURE 3 HERE $>$}

These diagnostic graphs provide evidence that the importance sampler is unreliable as they indicate a maximum likelihood estimate of $\xi$ which is larger than 0.5 . The variance of the importance weights does not exist for the SV-N model. This result is in line with the more informal assessment given in Figure 3(iii). This records the recursive variance estimator, which contains several large jumps. Standard asymptotics to measure the uncertainty of the importance sampler's estimate of the log-likelihood function is problematic in this case.

We now turn our attention to the empirically more interesting SV-t model and look at the diagnostic graphs of the model with estimated parameters. The estimated parameter values are provided in Table 3 and show a large improvement in the fit of the model over the full sample. Figure 4 presents the same diagnostic graphs as in Figure 3. It is clear that these graphs indicate a better behaved importance sampler. In particular, the estimated shape parameters provide 
evidence that two moments exist for the importance weights. When these diagnostic graphs are compared with the same graphs for the estimated SV- $N$ model, we conclude that the importance sampler for the SV-t model is more reliable.

\section{$<$ INSERT FIGURE 4 HERE $>$}

In Table 4, we present the test statistics for both SV models. The test statistics are computed for two different thresholds where we have chosen $u$ so that we have the 10,000 and 40,000 largest weights, respectively. The score and $t$-value statistics are signed tests for the null hypothesis $H_{0}: \xi=\frac{1}{2}$. We suspect that no variance exists for the importance weights when these tests have significant positive values. Both tests are asymptotically standard normally distributed, so having a $95 \%$ critical value of 1.64 . The $L R$ test follows an asymptotic $0.5\left(\chi_{0}^{2}+\chi_{1}^{2}\right)$ distribution with the $95 \%$ critical value 2.69 .

\section{$<$ INSERT TABLE 4 HERE $>$}

The results given in Table 4 are consistent across the choice of statistic. For the SV- $N$ model, the results are poor with all the statistics strongly rejecting the existence of a variance for the full sample. As we reduce the number of observations and the dimension of the integral decreases, the importance function for the SV- $N$ begins to perform better. Table 4 also includes test statistics computed from models estimated on roughly one-seventh the sample size (818 observations). At this point, the test statistics fail to reject the null hypothesis. Conversely, the test statistics indicate that the importance function for the SV- $t$ model has a finite variance for all sample sizes.

The point estimates in Table 3 appear reasonable for the SV- $N$ model, despite the fact that the weight function has an infinite variance. We believe users should still proceed with caution. Robert and Casella (2004, p. 95) emphasize that when the variance does not exist the range of Monte Carlo estimates computed using different sequences of random numbers will be enormous. For an SV model, this means that the estimated log-volatilities may be far from the true, unobserved values. Robert (2007) demonstrates how an importance sampler with a poorly chosen importance density can entirely miss-estimate the true log-volatilities for many sequences of random numbers.

\section{Conclusion}

Importance sampling is increasingly used in econometrics to estimate likelihoods and to compute moments of the posterior distribution in Bayesian analyses. Behind its use lies the assumption that the variance of the importance sampling weights exists. In this paper, we have proposed test 
statistics and graphical diagnostics to check this assumption in high dimensional settings when it is often difficult to evaluate analytically. We have applied our new tests on empirical problems and find this assumption is often overturned. This suggests that this is an important problem of which researchers should be aware. We hope that our methods will provide researchers with a better understanding of when their importance samplers are reliable.

\section{Acknowledgements}

We would like to thank the Editor and three referees for their insightful comments. Furthermore, we thank Shimrit Abraham for excellent computational support and Charles S. Bos for helpful discussions. All the computations reported in this paper were carried out using the OxMetrix 5.0 programming environment of Doornik (2007).

\section{References}

An, S. and F. Schorfheide (2007). Bayesian analysis of DSGE models. Econometric Reviews 26, 113-172.

Asai, M. and M. McAleer (2006). Asymmetric multivariate stochastic volatility. Econometric Reviews 25, 453-473.

Brandt, M. W. and Q. Kang (2004). On the relationship between the conditional mean and volatility of stock returns: a latent VAR approach. Journal of Financial Economics 72, $217-257$.

Cappé, O., E. Moulines, and T. Rydén (2005). Inference in Hidden Markov Models. New York: Springer Press.

Chernoff, H. (1954). On the distribution of the likelihood ratio. Annals of Mathematical Statistics $25,573-8$.

Cox, D. R. and D. V. Hinkley (1974). Theoretical Statistics. London: Chapman \& Hall.

Danielsson, J. (1994). Stochastic volatility in asset prices: estimation with simulated maximum likelihood. Journal of Econometrics 61, 375-400.

Danielsson, J. and C. G. de Vries (1997). Tail index and quantile estimation with very high frequency data. Journal of Empirical Finance 4, 241-257.

Danielsson, J. and J. F. Richard (1993). Accelerated Gaussian importance sampler with application to dynamic latent variable models. Journal of Applied Econometrics 8, S153-S174. 
Davison, A. C. and R. L. Smith (1990). Models for exceedances over high thresholds (with discussion). Journal of the Royal Statistical Society, Series B 52, 393-442.

Doornik, J. A. (2007). Ox: An Object-Oriented Matrix Programming Language. London: Timberlake Consultants Press.

Durbin, J. and S. J. Koopman (1997). Monte Carlo maximum likelihood estimation of nonGaussian state space models. Biometrika 84, 669-684.

Durham, G. (2003). Likelihood-based specification analysis of continuous-time models of the short-term interest rate. Journal of Financial Economics 70, 463-487.

Durham, G. (2006). Monte Carlo methods for estimating, smoothing, and filtering one- and two-factor stochastic volatility models. Journal of Econometrics 133, 273-305.

Durham, G. and A. R. Gallant (2002). Numerical techniques for maximum likelihood estimation of continuous-time diffusion processes (with discussion). Journal of Business and Economic Statistics 20, 297-338.

Elerian, O., S. Chib, and N. Shephard (2001). Likelihood inference for discretely observed non-linear diffusions. Econometrica 69, 959-993.

Embrechts, P., C. Kluppelberg, and T. Mikosch (1997). Modelling Extremal Events for Insurance and Finance. Berlin: Springer.

Geweke, J. (1989). Bayesian inference in econometric models using Monte Carlo integration. Econometrica 5\%, 1317-39.

Geweke, J. (2005). Contemporary Bayesian Econometrics and Statistics. New Jersey: Wiley Press.

Ghysels, E., A. C. Harvey, and E. Renault (1996). Stochastic volatility. In C. R. Rao and G. S. Maddala (Eds.), Statistical Methods in Finance, pp. 119-191. Amsterdam: North-Holland.

Gonzalo, J. and J. Olmo (2004). Which extreme values are really extreme? Journal of Financial Econometrics 2, 349-369.

Gourieroux, C., A. Holly, and A. Monfort (1981). Kuhn-Tucker, likelihood ratio and Wald tests for nonlinear models with inequality constraints on the parameters. Harvard Institute of Economic Research Discussion Paper 770.

Haeusler, E. and J. L. Teugels (1985). On asymptotic normality of Hill's estimator for the exponent of regular variation. Annals of Statistics 13, 743-756.

Hammersley, J. M. and D. C. Handscomb (1964). Monte Carlo Methods. London: Methuen and Co. 
Hendry, D. F. and J. F. Richard (1991). Likelihood evaluation for dynamic latent variable models. In H. M. Amman, D. A. Delsley, and L. F. Pau (Eds.), Computational Economics and Econometrics, pp. 3-17. Dordrecht: Kluwer.

Hill, B. (1975). A simple general approach to inference about the tail of a distribution. Annals of Statistics 3, 1163-1174.

Hull, J. and A. White (1987). The pricing of options on assets with stochastic volatilities. Journal of Finance 42, 281-300.

Jungbacker, B. and S. J. Koopman (2007). Monte Carlo estimation for nonlinear non-Gaussian state space models. Biometrika 94(4), 827-839.

Kahn, H. and A. W. Marshall (1953). Methods of reducing sample size in Monte Carlo computations. Journal of the Operational Research Society of America 1, 263-271.

Kloek, T. and H. K. van Dijk (1978). Bayesian estimates of equation system parameters: an application of integration by Monte Carlo. Econometrica 46, 1-20.

Liesenfeld, R. and J.-F. Richard (2003). Univariate and multivariate stochastic volatility models: estimation and diagnostics. Journal of Empirical Finance 10, 505-531.

Liu, J. S. (2001). Monte Carlo Strategies in Scientific Computing. New York: Springer Press.

Marshall, A. W. (1956). The use of multi-stage sampling schemes in Monte Carlo computations. In M. Meyer (Ed.), Symposium on Monte Carlo Methods, pp. 123-140. New York: Wiley.

Monahan, J. F. (1993). Testing the behaviour of importance sampling weights. Computer Science and Statistics: Proceedings of the 25th Annual Symposium on the Interface, 112117.

Monahan, J. F. (2001). Numerical Methods of Statistics. Cambridge: Cambridge University Press.

Pickands, J. (1975). Statistical inference using extreme order statistics. Annals of Statistics 3, $119-131$.

Richard, J. F. and W. Zhang (2007). Efficient high-dimensional importance sampling. Journal of Econometrics 141(2), 1385-1411.

Robert, C. P. (2007). Stochastic volatility: an experimental approach. CREST ENSAE, Paris, France.

Robert, C. P. and G. Casella (2004). Monte Carlo Statistical Methods (Second ed.). New York: Springer Press. 
Sandmann, G. and S. J. Koopman (1998). Estimation of stochastic volatility models via Monte Carlo maximum likelihood. Journal of Econometrics 87, 271-301.

Shephard, N. (Ed.) (2005). Stochastic Volatility: Selected Readings. Oxford: Oxford University Press.

Shephard, N. and M. K. Pitt (1997). Likelihood analysis of non-Gaussian measurement time series. Biometrika 84, 653-667.

Smith, R. L. (1985). Maximum likelihood estimation in a class of non-regular cases. Biometrika 72, 67-90.

Smith, R. L. (1987). Estimating tails of probability distributions. Annals of Statistics 15, $1174-1207$. 


\begin{tabular}{|c|c|c|c|c|c|c|c|c|c|c|}
\hline & \multicolumn{5}{|c|}{$N=10,000$} & \multicolumn{5}{|c|}{$N=100,000$} \\
\hline & score & $t$ & $L R$ & $M_{\text {test }}$ & $M_{\text {test }}^{*}$ & score & $t$ & $L R$ & $M_{\text {test }}$ & $M_{\text {test }}^{*}$ \\
\hline $\bar{~} u=0.55 \mathrm{~N}$ & & & & & & & & & & \\
\hline$\epsilon=0.5$ & 0.00 & 0.00 & 0.00 & 0.00 & 0.00 & 0.00 & 0.00 & 0.00 & 0.00 & 0.00 \\
\hline$\epsilon=0.8$ & 0.00 & 0.46 & 0.07 & 0.00 & 0.00 & 0.00 & 0.60 & 0.21 & 0.00 & 0.00 \\
\hline$\epsilon=1.0$ & 0.13 & 0.97 & 0.76 & 0.01 & 0.02 & 1.00 & 1.00 & 1.00 & 0.00 & 0.00 \\
\hline$\epsilon=1.2$ & 0.71 & 1.00 & 0.99 & 0.05 & 0.06 & 1.00 & 1.00 & 1.00 & 0.08 & 0.08 \\
\hline$\epsilon=1.5$ & 0.99 & 1.00 & 1.00 & 0.23 & 0.19 & 1.00 & 1.00 & 1.00 & 0.46 & 0.33 \\
\hline$\epsilon=3.0$ & 1.00 & 1.00 & 1.00 & 0.90 & 0.73 & 1.00 & 1.00 & 1.00 & 1.00 & 0.95 \\
\hline$\epsilon=5.0$ & 1.00 & 1.00 & 1.00 & 0.98 & 0.89 & 1.00 & 1.00 & 1.00 & 1.00 & 1.00 \\
\hline$u=0.50 N$ & & & & & & & & & & \\
\hline$\epsilon=0.5$ & 0.00 & 0.00 & 0.00 & - & - & 0.00 & 0.00 & 0.00 & - & - \\
\hline$\epsilon=0.8$ & 0.00 & 0.19 & 0.01 & - & - & 0.00 & 0.02 & 0.00 & - & - \\
\hline$\epsilon=1.0$ & 0.03 & 0.87 & 0.47 & - & - & 0.93 & 1.00 & 0.99 & - & - \\
\hline$\epsilon=1.2$ & 0.41 & 1.00 & 0.94 & - & - & 1.00 & 1.00 & 1.00 & - & - \\
\hline$\epsilon=1.5$ & 0.95 & 1.00 & 1.00 & - & - & 1.00 & 1.00 & 1.00 & - & - \\
\hline$\epsilon=3.0$ & 1.00 & 1.00 & 1.00 & - & - & 1.00 & 1.00 & 1.00 & - & - \\
\hline$\epsilon=5.0$ & 1.00 & 1.00 & 1.00 & - & - & 1.00 & 1.00 & 1.00 & - & - \\
\hline$u=0.40 N$ & & & & & & & & & & \\
\hline$\epsilon=0.8$ & 0.00 & 0.02 & 0.00 & - & - & 0.00 & 0.00 & 0.00 & - & - \\
\hline$\epsilon=1.0$ & 0.00 & 0.48 & 0.11 & - & - & 0.02 & 0.83 & 0.42 & - & - \\
\hline$\epsilon=1.2$ & 0.08 & 0.92 & 0.63 & - & - & 0.99 & 1.00 & 1.00 & - & - \\
\hline$\epsilon=1.5$ & 0.64 & 1.00 & 0.98 & - & - & 1.00 & 1.00 & 1.00 & - & - \\
\hline$\epsilon=3.0$ & 0.82 & 1.00 & 1.00 & - & - & 1.00 & 1.00 & 1.00 & - & - \\
\hline$\epsilon=5.0$ & 0.97 & 1.00 & 1.00 & - & - & 1.00 & 1.00 & 1.00 & - & - \\
\hline$u=0.30 N$ & & & & & & & & & & \\
\hline$\epsilon=0.8$ & 0.00 & 0.01 & 0.00 & - & - & 0.00 & 0.00 & 0.00 & - & - \\
\hline$\epsilon=1.0$ & 0.00 & 0.20 & 0.03 & - & - & 0.00 & 0.10 & 0.01 & - & - \\
\hline$\epsilon=1.2$ & 0.01 & 0.68 & 0.30 & - & - & 0.55 & 0.99 & 0.97 & - & - \\
\hline$\epsilon=1.5$ & 0.28 & 0.97 & 0.85 & - & - & 1.00 & 1.00 & 1.00 & - & - \\
\hline$\epsilon=3.0$ & 0.91 & 1.00 & 0.98 & - & - & 1.00 & 1.00 & 1.00 & - & - \\
\hline$\epsilon=5.0$ & 1.00 & 1.00 & 1.00 & - & - & 1.00 & 1.00 & 1.00 & - & - \\
\hline$u=0.10 N$ & & & & & & & & & & \\
\hline$\epsilon=0.8$ & 0.00 & 0.01 & 0.00 & - & - & 0.00 & 0.00 & 0.00 & - & - \\
\hline$\epsilon=1.0$ & 0.00 & 0.05 & 0.01 & - & - & 0.00 & 0.00 & 0.00 & - & - \\
\hline$\epsilon=1.2$ & 0.00 & 0.19 & 0.08 & - & - & 0.01 & 0.37 & 0.22 & - & - \\
\hline$\epsilon=1.5$ & 0.03 & 0.47 & 0.34 & - & - & 0.72 & 0.98 & 0.99 & - & - \\
\hline$\epsilon=3.0$ & 0.75 & 0.98 & 0.76 & - & - & 1.00 & 1.00 & 1.00 & - & - \\
\hline$\epsilon=5.0$ & 0.97 & 1.00 & 1.00 & - & - & 1.00 & 1.00 & 1.00 & - & - \\
\hline$u=0.01 N$ & $\bar{T}$ & & & & & & & & & \\
\hline$\epsilon=0.8$ & 0.00 & 0.01 & 0.01 & - & - & 0.00 & 0.00 & 0.00 & - & - \\
\hline$\epsilon=1.0$ & 0.00 & 0.01 & 0.02 & - & - & 0.00 & 0.00 & 0.01 & - & - \\
\hline$\epsilon=1.2$ & 0.00 & 0.00 & 0.05 & - & - & 0.00 & 0.00 & 0.08 & - & - \\
\hline$\epsilon=1.5$ & 0.01 & 0.00 & 0.10 & - & - & 0.04 & 0.00 & 0.37 & - & - \\
\hline$\epsilon=3.0$ & 0.08 & 0.00 & 0.34 & - & - & 0.81 & 0.01 & 0.99 & - & - \\
\hline$\epsilon=5.0$ & 0.17 & 0.00 & 0.51 & - & - & 0.98 & 0.02 & 1.00 & - & - \\
\hline
\end{tabular}

Table 1: Normal density experiment. Monte Carlo study for power and size with size $\alpha=0.05$. Percentage of rejections of the null hypothesis computed from 10,000 Monte Carlo replications. The $M_{\text {test }}$ used $k=4 N^{1 / 3}$ while $M_{\text {test }}^{*}$ used $k=2 N^{1 / 3}$. 


\begin{tabular}{l|ccccc|ccccr} 
& \multicolumn{7}{|c|}{$N=10,000$} & \multicolumn{7}{c}{$N=100,000$} \\
\hline & score & $t$ & $L R$ & $M_{\text {test }}$ & $M_{\text {test }}^{*}$ & score & $t$ & $L R$ & $M_{\text {test }}$ & $M_{\text {test }}^{*}$ \\
\hline \hline$u=0.50 N$ & & & & & & & & & & \\
$\nu=1$ & 1.00 & 1.00 & 1.00 & 0.97 & 0.88 & 1.00 & 1.00 & 1.00 & 1.00 & 1.00 \\
$\nu=2$ & 1.00 & 1.00 & 1.00 & 0.87 & 0.75 & 1.00 & 1.00 & 1.00 & 1.00 & 0.99 \\
$\nu=5$ & 1.00 & 1.00 & 1.00 & 0.19 & 0.26 & 1.00 & 1.00 & 1.00 & 0.84 & 0.76 \\
$\nu=20$ & 1.00 & 1.00 & 1.00 & 0.00 & 0.00 & 1.00 & 1.00 & 1.00 & 0.00 & 0.00 \\
$\nu=100$ & 1.00 & 1.00 & 1.00 & 0.00 & 0.00 & 1.00 & 1.00 & 1.00 & 0.00 & 0.00 \\
\hline$u=0.10 N$ & & & & & & & & & & \\
$\nu=1$ & 0.98 & 1.00 & 1.00 & - & - & 1.00 & 1.00 & 1.00 & - & - \\
$\nu$ & 0.89 & 1.00 & 1.00 & - & - & 1.00 & 1.00 & 1.00 & - & - \\
$\nu=5$ & 0.37 & 1.00 & 0.87 & - & - & 1.00 & 1.00 & 1.00 & - & - \\
$\nu=20$ & 0.00 & 0.50 & 0.05 & - & - & 0.00 & 0.46 & 0.06 & - & - \\
$\nu=100$ & 0.00 & 0.00 & 0.00 & - & - & 0.00 & 0.00 & 0.00 & - & - \\
\hline$u=0.01 N$ & & & & & & & & & & \\
$\nu=1$ & 0.21 & 0.02 & 0.56 & - & - & 1.00 & 0.90 & 1.00 & - & - \\
$\nu=2$ & 0.15 & 0.05 & 0.47 & - & - & 0.97 & 0.84 & 1.00 & - & - \\
$\nu=5$ & 0.04 & 0.15 & 0.24 & - & - & 0.49 & 0.78 & 0.91 & - & - \\
$\nu=20$ & 0.00 & 0.14 & 0.01 & - & - & 0.00 & 0.02 & 0.00 & - & - \\
$\nu=100$ & 0.00 & 0.01 & 0.00 & - & - & 0.00 & 0.00 & 0.00 & - & - \\
\hline
\end{tabular}

Table 2: Normal approximation to the Student's $t$ experiment. Monte Carlo study for power with size $\alpha=0.05$. Percentage of rejections of the null hypothesis computed from 10, 000 Monte Carlo replications. The $M_{\text {test }}$ used $k=4 N^{1 / 3}$ while $M_{\text {test }}^{*}$ used $k=2 N^{1 / 3}$. 
Stochastic volatility model

\begin{tabular}{|c|c|c|c|c|}
\hline & \multicolumn{2}{|c|}{ full sample } & \multicolumn{2}{|c|}{$1 / 3$ sample } \\
\hline & $\mathrm{SV}-N$ & SV- $t$ & $\mathrm{SV}-N$ & SV- $t$ \\
\hline \multirow[t]{2}{*}{$\mu_{y}$} & 0.0662 & 0.0636 & 0.0472 & 0.0481 \\
\hline & $\left(\begin{array}{ll}0.0470 & 0.0854\end{array}\right)$ & $\left(\begin{array}{ll}0.0448 & 0.0825\end{array}\right)$ & $\left(\begin{array}{ll}0.0090 & 0.0854\end{array}\right)$ & $\left(\begin{array}{ll}0.0097 & 0.0864\end{array}\right)$ \\
\hline \multirow[t]{2}{*}{$\phi$} & 0.983 & 0.993 & 0.993 & 0.994 \\
\hline & $(0.978 \quad 0.987)$ & $(0.988 \quad 0.996)$ & $\left(\begin{array}{ll}0.981 & 0.997\end{array}\right)$ & $\left(\begin{array}{ll}0.981 & 0.998\end{array}\right)$ \\
\hline \multirow[t]{2}{*}{$\sigma_{\eta}$} & 0.0226 & 0.0077 & 0.0088 & 0.007 \\
\hline & $\left(\begin{array}{ll}0.0183 & 0.0278\end{array}\right)$ & $\left(\begin{array}{ll}0.0050 & 0.0120\end{array}\right)$ & $\left(\begin{array}{ll}0.0044 & 0.0177\end{array}\right)$ & $\left(\begin{array}{ll}0.0033 & 0.0161\end{array}\right)$ \\
\hline \multirow[t]{2}{*}{$\mu_{\alpha}$} & -0.367 & -0.309 & -0.434 & -0.402 \\
\hline & $\left(\begin{array}{ll}-0.600 & -0.135\end{array}\right)$ & $\left(\begin{array}{ll}-0.663 & 0.0459\end{array}\right)$ & $\left(\begin{array}{ll}-1.125 & 0.258\end{array}\right)$ & $\left(\begin{array}{ll}-1.258 & 0.454\end{array}\right)$ \\
\hline$\nu$ & - & 7.79 & - & 24.68 \\
\hline log-like & $-7,270.0$ & .222 .8 & $-1,743.7$ & -1.742 .4 \\
\hline & & & & \\
\hline
\end{tabular}

Table 3: Simulated maximum likelihood estimates of the parameters of the two SV models. The values in parentheses give the asymptotic (asymmetric) $95 \%$ confidence intervals. 


\begin{tabular}{|c|c|c|c|c|c|c|}
\hline & \multicolumn{6}{|c|}{ Stochastic volatility model } \\
\hline & \multicolumn{2}{|c|}{ full sample } & \multicolumn{2}{|c|}{$1 / 3$ sample } & \multicolumn{2}{|c|}{$1 / 7$ sample } \\
\hline & $\mathrm{SV}-N$ & $\mathrm{SV}-t$ & $\mathrm{SV}-N$ & $\mathrm{SV}-t$ & $\mathrm{SV}-N$ & SV- $t$ \\
\hline \multicolumn{7}{|c|}{$u=0.4 N$} \\
\hline score & 128.44 & -10.42 & 17.09 & -16.87 & -0.252 & -21.80 \\
\hline$t$ & 1810.0 & -27.29 & 44.44 & -60.42 & -0.577 & -118.5 \\
\hline$L R$ & 20464.1 & 0 & 1078.0 & 0 & 0 & 0 \\
\hline$M_{\text {test }}$ & 20.01 & -3.83 & 0.061 & -5.65 & 0.232 & -8.15 \\
\hline \multicolumn{7}{|c|}{$u=0.1 N$} \\
\hline score & 38.33 & -5.52 & 3.68 & -7.42 & -0.825 & -9.49 \\
\hline$t$ & 59.51 & -9.28 & 3.22 & -21.01 & -0.930 & -49.01 \\
\hline$L R$ & 2755.6 & 0 & 57.46 & 0 & 0 & 0 \\
\hline
\end{tabular}

Table 4: Likelihood based tests for the null of the existence of the variance of the importance weights for two SV models estimated by simulated maximum likelihood. The $95 \%$ critical values for the score, $t, L R$, and $M_{\text {test }}$ are 1.65, 1.65, 2.69, and 1.65 respectively. The $M_{\text {test }}$ was computed using $k=4 N^{1 / 3}$. 

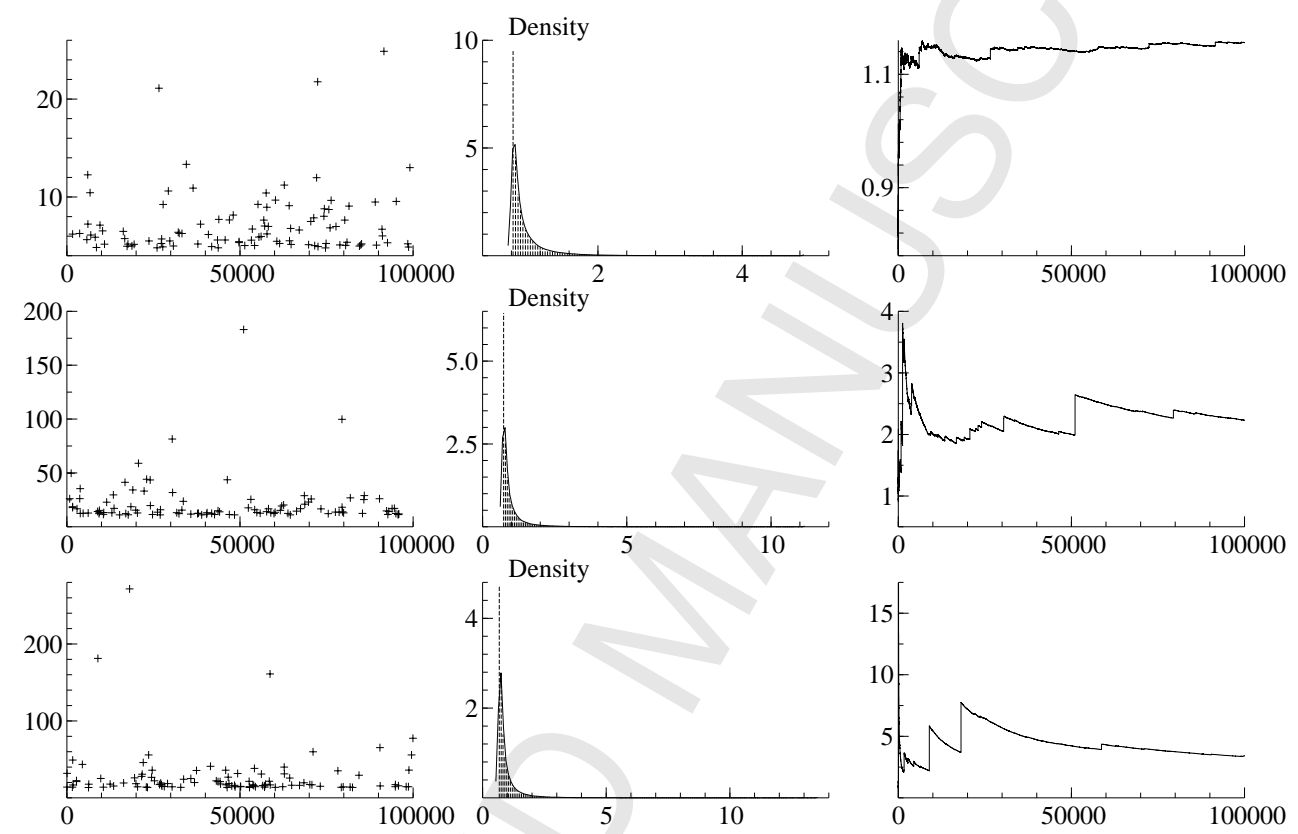

Figure 1: Graphical diagnostics for the normal experiment. Top, middle, and bottom rows represent $\epsilon=0.5,1.0,1.2$ respectively. The left hand side pictures are of the largest 100 weights, the middle pictures are a histogram of the remaining weights and the right hand side shows a recursive estimator of the variance of the weights. $N=100,000$ draws. 

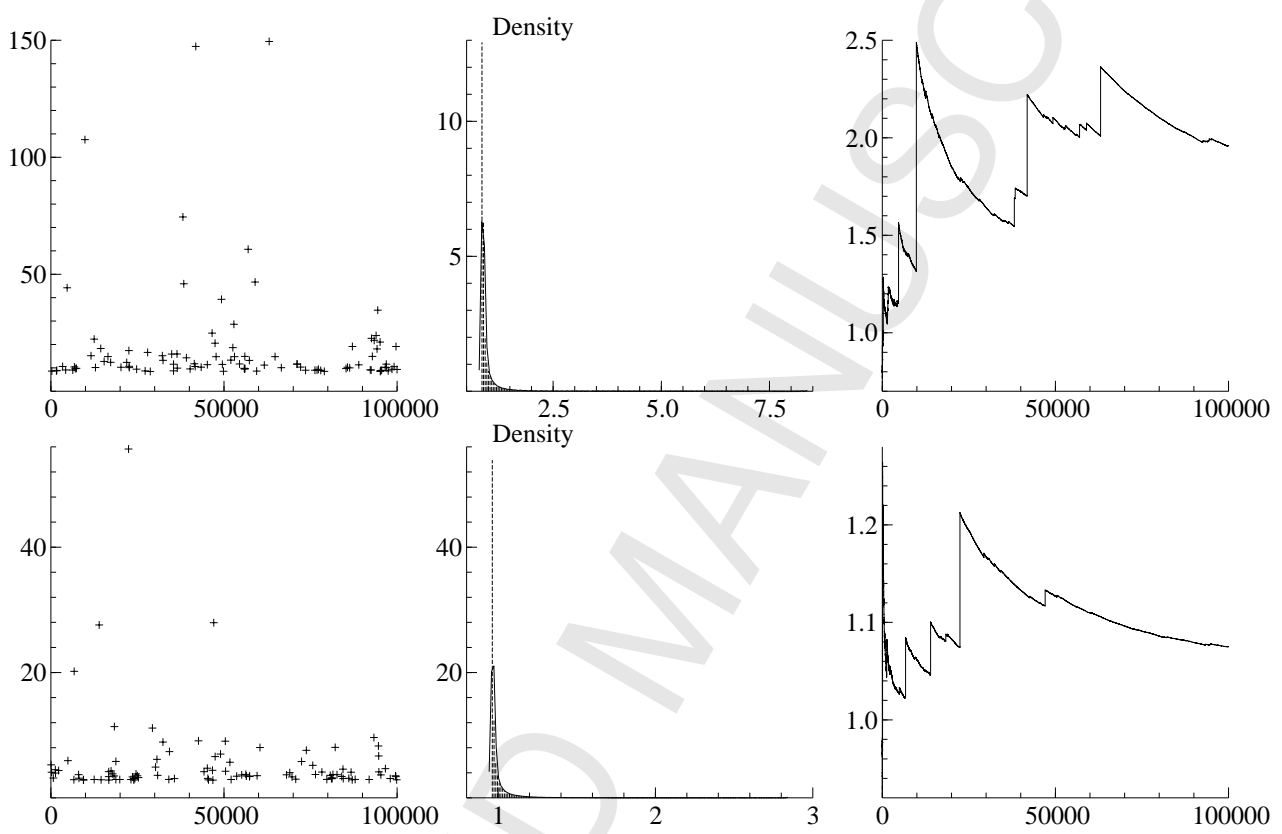

Figure 2: Graphical diagnostics for the Student's $t / n o r m a l$ experiment. Top and bottom rows represent $\nu=5$ and 20, respectively. The left hand side pictures are of the largest 100 weights, the middle pictures are a histogram of the remaining weights and the right hand side shows a recursive estimator of the variance of the weights. $N=100,000$ draws. 

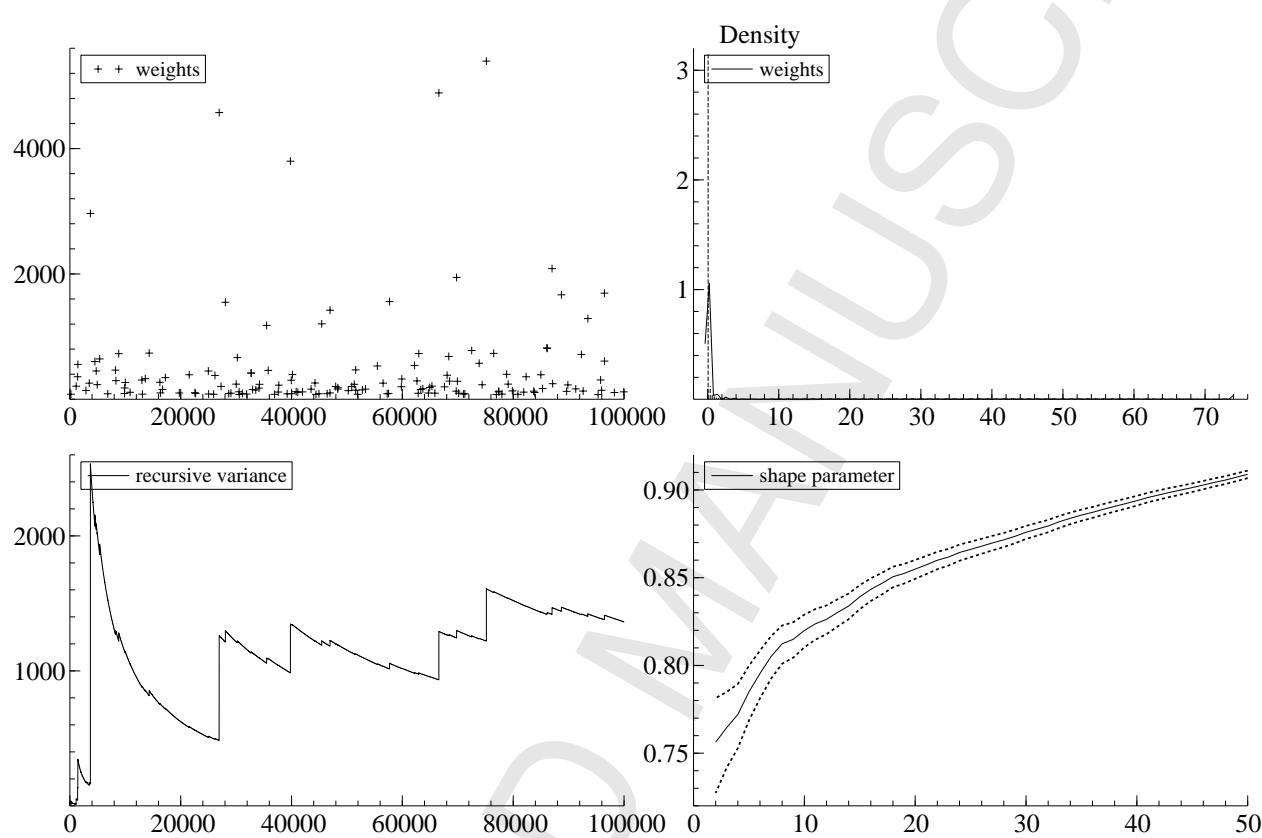

Figure 3: Importance sampling diagnostic graphics for the $S V-N$ model. (i) Largest $100 \mathrm{im}$ portance weights; (ii) histogram of the remaining weights; (iii) recursive variance estimator of the weights; (iv) estimated shape parameters with $95 \%$ confidence intervals (dotted lines) for 50 different thresholds going from the largest $1 \%$ to $50 \%$ of the $\mathrm{N}^{+}$weights. 

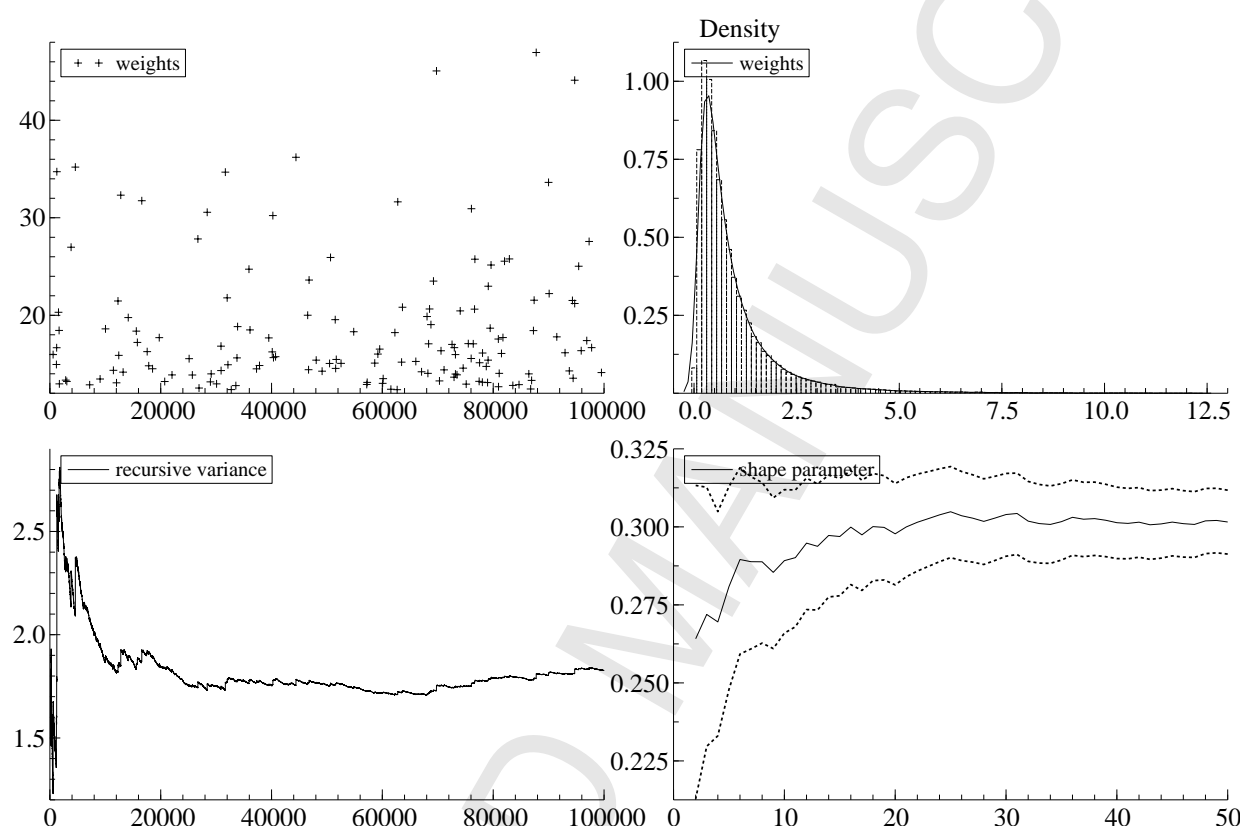

Figure 4: Importance sampling diagnostic graphics for the $S V$-t model. (i) Largest 100 importance weights; (ii) histogram of the remaining weights; (iii) recursive variance estimator of the weights; (iv) estimated shape parameters with $95 \%$ confidence intervals (dotted lines) for 50 different thresholds going from the largest $1 \%$ to $50 \%$ of the $\mathrm{N}^{+}$weights. 